\title{
Potencial do Uso da Restrição Hídrica em Testes de Sanidade de Sementes de Algodoeiro
}

\author{
Andréia Q. Machado', José da Cruz Machado², Maria D.G.G.C. Vieira ${ }^{3}$, \\ Daniel Cassetari Neto ${ }^{4} \&$ Marcella V. Souza ${ }^{2}$
}

${ }^{1}$ Laboratório de Fitopatologia, GPA de Ciências Agrárias e Biológicas, Centro Universitário Univag, CEP 78118-000, Várzea Grande, MT, Brasil, e-mail: machadoaq@terra.com.br; ${ }^{2}$ Departamento de Fitopatologia, ${ }^{3}$ Departamento de Fitotecnia, Universidade Federal de Lavras, Cx. Postal 3037, CEP 37200-000, Lavras, MG, Brasil; ${ }^{4}$ Departamento de Fitotecnia e Fitossanidade, Faculdade de Agronomia e Medicina Veterinária, Universidade Federal de Mato Grosso, CEP 78060-900, Cuiabá, MT, Brasil.

Autor para correspondência: Andréia Quixabeira Machado

MACHADO, A.Q., MACHADO, J.C., VIEIRA, M.D.G.G.C., CASSETARI NETO, D. \& SOUZA, M.V. Potencial do uso da restrição hídrica em testes de sanidade de sementes de algodoeiro. Fitopatologia Brasileira 32:408-414. 2007.

\section{RESUMO}

Nos testes de sanidade, realizados por meio dos métodos de incubação em papel de filtro e em meio agarizado, a germinação rápida das sementes dificulta a identificação dos fungos e pode comprometer a validade desses métodos. Para impedir ou reduzir a germinação de sementes de espécies dicotiledôneas, o uso de 2,4-D (sal de sódio) tem sido a alternativa disponível havendo, no entanto, questionamentos sobre este composto por inúmeras razões, inclusive o aspecto toxicológico. Neste trabalho, o objetivo foi estudar a viabilidade do uso da restrição hídrica como recurso para reduzir a germinação das sementes em substituição ao 2,4-D. Foram utilizados os restritores hídricos manitol e Cloreto de sódio, nos potenciais - $0,6,-0,8,-1,0$ e -1,2 $\mathrm{MPa}$, sendo avaliados seus efeitos sobre a germinação das sementes, comprimento de plântulas e crescimento micelial in vitro dos principais fungos associados a sementes de algodoeiro e seu desenvolvimento sobre as sementes no teste de incubação em papel de filtro. A restrição hídrica proporcionada pelos solutos e potenciais osmóticos testados foi capaz de reduzir a germinação e o comprimento das plântulas em níveis considerados satisfatórios para a análise sanitária de sementes de algodoeiro, além de não interferir na recuperação dos principais fungos sobre as sementes no teste de papel de filtro, revelando-se desta forma como uma alternativa eficaz em substituição ao uso do 2,4-D. O crescimento micelial dos fungos em meio agarizado foi reduzido nos potenciais osmóticos a partir de -0,6 MPa. A recuperação dos fungos fitopatogênicos através do teste de incubação em papel de filtro não foi afetada pelo método da restrição hídrica.

Palavras-chave adicionais: patologia de sementes, fungos.

\begin{abstract}
Potential of water restriction use in cotton seed health testing

The fast germination of cotton seeds that occurs in blotter and agar seed health tests may become a difficulty in the identification of fungi, thus affecting the efficiency of these tests. The available alternative to impede or to reduce seed germination of dicotyledoneous species is the incorporation of 2.4-D (sodium salt formulation) solution to the substrate. Nevertheless, some questions about 2,4-D use may be raised for several reasons, including the toxicity of this compound. The aim of this work was to investigate the viability of the use of water restriction technique in place of the 2,4-D method to reduce seed germination in incubation seed health testing. The effects of water restriction produced by mannitol and sodium chloride at $-0,6,-0,8,-1,0$ and $-1,2 \mathrm{MPa}$ osmotic potentials were evaluated on seed germination, seedling elongation and in vitro mycelial growth of some fungi occurring on cotton seeds and on their development in the blotter test. The water restriction provided by the solutes at the osmotic potentials tested was proved to reduce germination and seedling elongation to satisfactory levels for cotton seed health analysis, allowing recovery of the main fungi on those seeds under blotter test conditions. This method was seen to be an efficient alternative to the use of 2,4-D. The mycelial growth of fungi was reduced on agar medium at osmotic potentials higher than $-0,6 \mathrm{MPa}$. The recovery of pathogenic fungi under blotter test was not affected by the water restriction technique.
\end{abstract}

Additional keywords: seed health testing, fungi.

\section{INTRODUÇÃO}

Patógenos que ocorrem em sementes de algodoeiro tais como Rhizoctonia solani J.G. Kühn, Colletotrichum gossypii

Parte da Dissertação de Mestrado do primeiro autor. Universidade Federal de Lavras. Lavras MG. 2002.
South., Colletotrichum gossypii var. cephalosporioides A.S. Costa, Fusarium spp., Fusarium oxysporum f.sp. vasinfectum W.C. Snyder \& H.N. Hansen, Botryodiplodia theobromae Pat., são responsáveis por grandes perdas na cultura no campo, com conseqüente elevação dos custos de produção, causando prejuízos dos mais elevados aos produtores (Pozza \& Juliatti, 1994; Juliatti \& Ruano, 1997; Cia \& Salgado, 1997; Cassetari Neto \& Machado, 2000). 
Nos testes de sanidade de sementes de algumas espécies vegetais realizados por meio dos métodos de incubação, seja em substrato de papel ou em meio agarizado, a germinação rápida das sementes tem sido um componente que dificulta o exame e possibilita a ocorrência de contaminações secundárias entre as sementes e o exterior do recipiente. $\mathrm{O}$ uso de 2,4-Diclorofenoxiacetato de sódio para inibir a germinação das sementes apresenta limitações não só por ser tóxico à saúde humana, mas também por ser fungitóxico em alguns casos (Limonard, 1968; Machado, 1980).

O uso da técnica de restrição hídrica, em substratos utilizados para inocular fungos em sementes, tem sido eficaz no controle (inibição) da germinação das sementes sem afetar o desenvolvimento dos fungos já testados (Amaral et al., 1996; Carvalho, 1999; Costa, 2000; Machado et al., 2004a). Este trabalho objetivou avaliar a viabilidade do uso da técnica de restrição hídrica no teste de sanidade para sementes de algodoeiro, como alternativa ao uso de 2,4-D.

\section{MATERIAL E MÉTODOS}

No primeiro ensaio foram avaliados os efeitos da restrição hídrica na germinação de sementes do algodoeiro, cultivar Coodetec 404, nas condições do teste de sanidade por meio dos métodos de incubação em substrato de papel e em meio BDA (batata-dextrose-ágar). Como restritores hídricos foram utilizadas soluções osmóticas dos solutos manitol e cloreto de sódio $(\mathrm{NaCl})$ nos potenciais de $-0,6$ $\mathrm{MPa},-0,8 \mathrm{MPa},-1,0 \mathrm{MPa}$ e -1,2 MPa, além das testemunhas água e BDA (-0,35 MPa). Também, para fins de comparação, utilizou-se o 2,4-D na concentração de $10 \mu \mathrm{g} \mathrm{x} \mathrm{mL} L^{-1}$. No final do período de incubação (sétimo dia), foram avaliados a porcentagem de germinação das sementes e o comprimento das plântulas $(\mathrm{cm})$. $\mathrm{O}$ ensaio constou de 10 tratamentos em delineamento inteiramente casualizado, com 4 repetições de 50 sementes, em esquema fatorial $2 \times 4+2$ (dois solutos, quatro potenciais osmóticos e dois tratamentos adicionais).

No segundo ensaio os efeitos da restrição hídrica em relação ao desenvolvimento dos fungos $R$. solani, $C$. gossypii, $C$. gossypii var. cephalosporioides, F. oxysporum f.sp. vasinfectum, B. theobromae e Sclerotinia sclerotiorum (Lib.) de Bary foram avaliados em meio ágar (BDA) por meio do índice de velocidade de crescimento micelial (IVCM) de todos os isolados utilizados. Para o cálculo deste índice, avaliações foram feitas diariamente durante o período de incubação, medindo-se o diâmetro médio das colônias e aplicando a fórmula utilizada por Oliveira (1991). O ensaio constou de 9 tratamentos em delineamento inteiramente casualizado, com 4 repetições (5 placas de Petri de $9 \mathrm{~cm}$ de diâmetro) em esquema fatorial $2 \times 4+1$ (dois solutos, quatro potenciais osmóticos e um tratamento adicional), e foi realizado separadamente para cada fungo avaliado.

No terceiro ensaio o efeito da restrição hídrica na ocorrência de fungos em sementes de algodoeiro foi avaliado utilizando-se sementes inoculadas com os fungos $C$. gossypii, C. gossypii var. cephalosporioides, F. oxysporum f.sp. vasinfectum e $B$. theobromae, em separado. Para a inoculação, lançou-se mão da técnica descrita por Machado et al. (2004a). Por esta técnica, as sementes foram mantidas em contato com as colônias fúngicas desenvolvidas em meio BDA contendo manitol, com potencial osmótico de $-1,0$ $\mathrm{MPa}$ em incubadora BOD à temperatura de $20^{\circ} \mathrm{C} \pm 1{ }^{\circ} \mathrm{C} \mathrm{e}$ fotoperíodo de 12 horas durante 5-7 dias.

A proporção de sementes inoculadas em relação às sementes não inoculadas para cada fungo foi de $25 \%$. As folhas de papel de filtro utilizadas no teste de sanidade (Incubação em substrato de papel) foram esterilizadas e umedecidas com as soluções de $\mathrm{NaCl}$ e manitol no potencial osmótico de-1,0 MPa (Machado et al., 2004b), e ainda 2,4-D na concentração de $10 \mu \mathrm{g} \cdot \mathrm{mL}^{-1}$. As sementes foram mantidas em câmara de incubação com temperatura de $20^{\circ} \mathrm{C} \pm 2^{\circ} \mathrm{C}$ e fotoperíodo de 12 horas durante sete dias. $\mathrm{O}$ ensaio constou de 3 tratamentos em delineamento inteiramente casualizado, com 4 repetições de 50 sementes (2 placas de Petri de $15 \mathrm{~cm}$ de diâmetro) para cada fungo estudado.

\section{RESULTADOS E DISCUSSÃO}

A porcentagem de germinação e o comprimento de plântulas referentes aos tratamentos com solução osmótica foram reduzidos substancialmente, evidenciando os efeitos da restrição hídrica em comparação com os tratamentos com água e 2,4-D (Tabela 1). Em relação à germinação, não houve diferença quanto aos efeitos dos solutos utilizados e em relação ao comprimento das plântulas verificou-se que com $\mathrm{NaCl}$, os valores foram significativamente menores ao manitol em substrato de papel.

No teste de incubação em $\mathrm{BDA}$, a maior redução da porcentagem de germinação foi verificada no potencial osmótico de -1,04 MPa (Figuras 1 e 2). Pelas equações de regressão linear e quadrática, verifica-se que a maior redução da porcentagem de germinação e do comprimento de plântulas no teste de incubação em substrato de papel ocorreu no potencial osmótico de $-1,2 \mathrm{MPa}$ para os dois solutos avaliados (Figuras 1 e 2).

A porcentagem de germinação e comprimento de plântulas no teste de incubação em substrato de papel apresentou valores inversos em relação aos potenciais osmóticos. Esses resultados evidenciam que a restrição hídrica, nestas condições, reduzindo a germinação das sementes torna-se extremamente útil no teste de incubação em substrato de papel, por dificultar a ocorrência de contaminações secundárias entre as sementes durante 0 período de incubação. Portanto, com a restrição hídrica, facilita-se o exame das sementes, possibilitando maior segurança na detecção dos fungos desenvolvidos sobre as mesmas.

As condições para o condicionamento osmótico de sementes variam amplamente em função das características de cada espécie e cultivar, e possivelmente, entre lotes de um mesmo cultivar. Por esses fatores, são verificadas diferenças 
TABELA 1 - Valores médios de porcentagem de germinação de sementes e comprimento de plântulas de algodoeiro submetidas ao teste de sanidade pelos métodos de incubação em papel de filtro e em BDA modificados pela adição dos solutos manitol e $\mathrm{NaCl}$ em diferentes níveis de potencial osmótico.

\begin{tabular}{|c|c|c|c|c|c|c|}
\hline \multirow{3}{*}{$\begin{array}{l}\text { Tratamentos } \\
\text { controle }^{1}\end{array}$} & \multicolumn{4}{|c|}{ Incubação em Papel de Filtro* } & \multirow{2}{*}{\multicolumn{2}{|c|}{$\frac{\text { Incubação em BDA* }}{\text { Germinação (\%) }}$}} \\
\hline & \multicolumn{2}{|c|}{ Germinação (\%) } & \multicolumn{2}{|c|}{ Comprimento de plântulas $(\mathrm{cm})$} & & \\
\hline & 95,00 & A & 5,01 & A & - & \\
\hline $2,4-\mathrm{D}$ & 84,75 & $\mathrm{~A}$ & 0,68 & $\mathrm{~B}$ & 66,25 & B \\
\hline controle $^{2}$ & - & & - & & 96,50 & A \\
\hline Média & 89,86 & $A$ & 2,85 & $a$ & 81,38 & $a$ \\
\hline Manitol & 63,13 & $\mathrm{~A}$ & 2,48 & $\mathrm{a}$ & 10,75 & $\mathrm{a}$ \\
\hline $\mathrm{NaCl}$ & 64,50 & $\mathrm{~A}$ & 1,74 & $\mathrm{~b}$ & 10,75 & $\mathrm{a}$ \\
\hline média & 63,82 & $B$ & 2,11 & $b$ & 10,75 & $b$ \\
\hline
\end{tabular}

* = comparação dos tratamentos nas colunas

$\mathrm{A}, \mathrm{B}=$ Teste de $\mathrm{F}$ significativo a $5 \%$ de probabilidade para tratamentos adicionais

$\mathrm{a}, \mathrm{b}=$ Teste de $\mathrm{F}$ significativo a $5 \%$ de probabilidade para os solutos

$a, b=$ Teste de $\mathrm{F}$ significativo a $5 \%$ de probabilidade para a interação fatorial $\mathrm{x}$ adicional

1 = água

$2=\mathrm{BDA}$

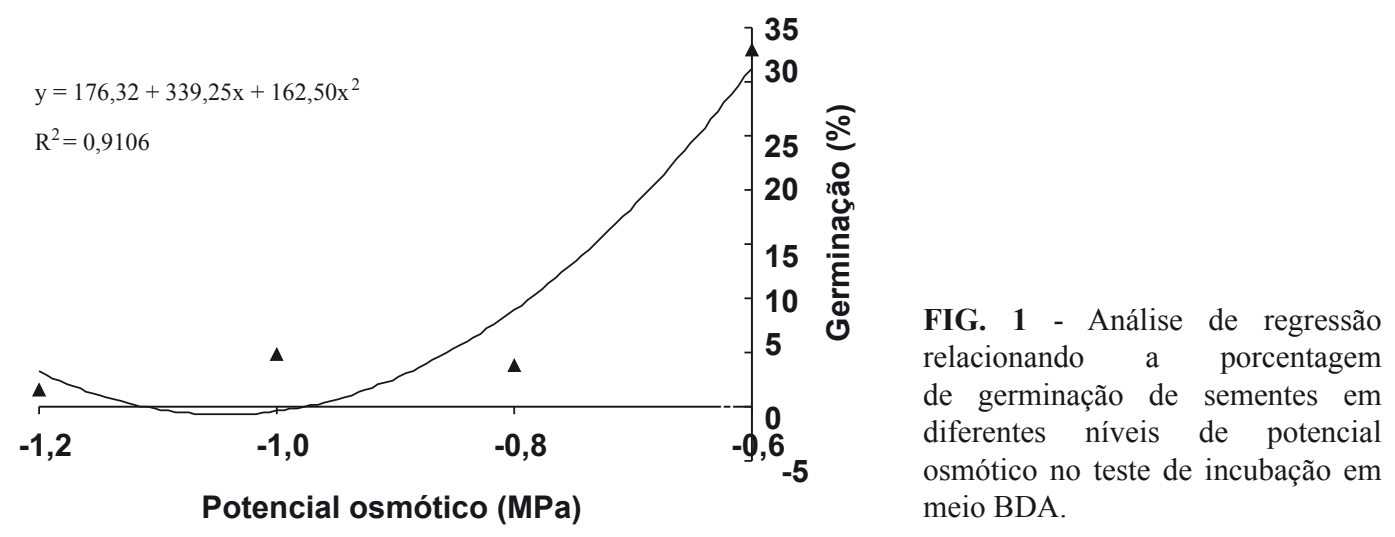

entre os solutos quanto à eficácia em reduzir e/ou retardar a germinação e o comprimento de plântulas (Heydecker et al., 1975).

Na Figura 3, verifica-se que para o IVCM, de $B$. theobromae, o maior valor foi obtido com o uso do soluto manitol no potencial osmótico de $-0,6 \mathrm{MPa}$, e, para $C$. gossypii var cephalosporioides, no potencial osmótico $-0,6$ MPa com o emprego de ambos os solutos, manitol e $\mathrm{NaCl}$, a maior redução desse último fungo ocorreu no potencial osmótico $-0,95 \mathrm{MPa}$ com o emprego do soluto $\mathrm{NaCl}$. Maiores valores de IVCM de Colletotrichum gossypii foram obtidos com o emprego do soluto $\mathrm{NaCl}$, potencial osmótico $-0,6 \mathrm{MPa}$ (Figura 4).

Para F. oxysporum f.sp. vasinfectum o IVCM foi maior na restrição hídrica promovida pelo soluto manitol e com base na regressão linear, o máximo aumento do IVCM ocorreu no potencial osmótico de -0,82 $\mathrm{MPa}$ (Figura 5). Para R. solani (Figura 4) e S. sclerotiorum (Figura 5), verificase que os maiores valores de IVCM ocorreram no potencial osmótico -0,6 MPa.

Em estudos sobre crescimento micelial de fungos em meios de cultura osmoticamente modificados com a adição de solutos iônicos e não-iônicos, vários pesquisadores têm constatado que esses organismos diferem na habilidade de absorver água do ambiente, e que existe uma faixa de potencial hídrico adequada ao crescimento de cada espécie (Alam et al, 1996; Carvalho, 1999; Coutinho, 2000; Costa, 2000).

Estudo realizado por Gao \& Shain (1995) com Cryphonectria parasitica (Murrill) M.E. Barr revelou que 


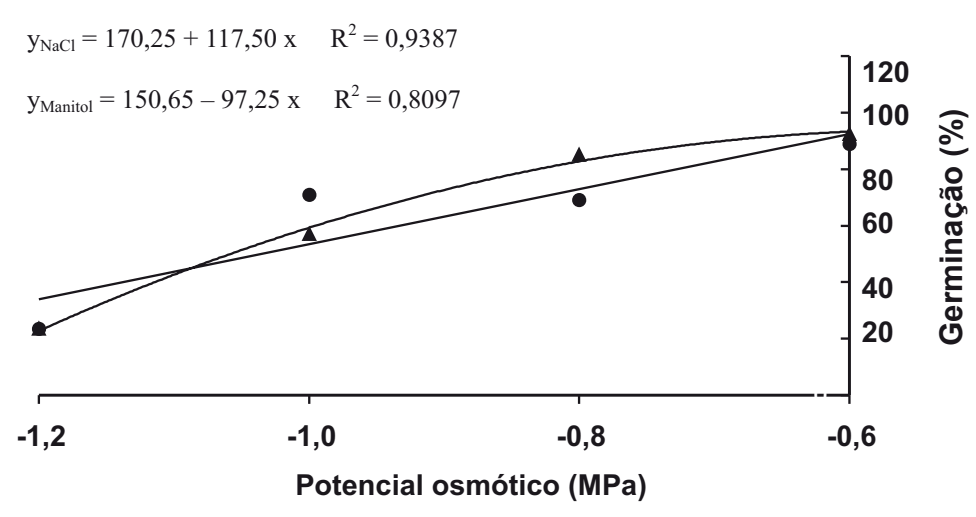

$\triangle \mathrm{NaCl} \bullet$ Manitol

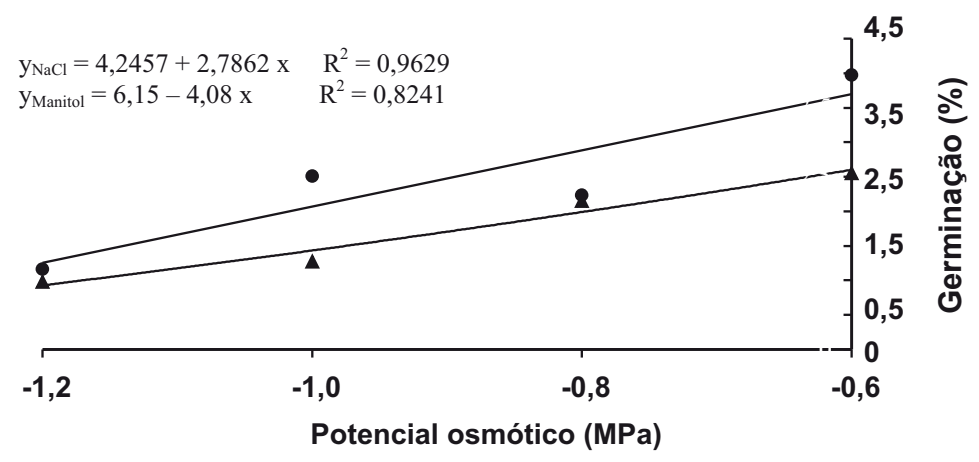

$\Delta \mathrm{NaCl} \bullet$ Manitol

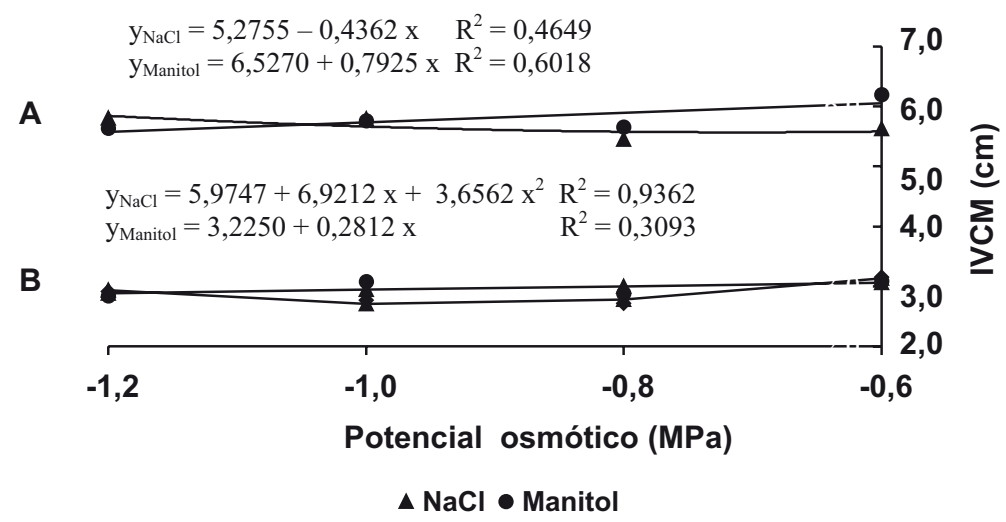

FIG. 2 - Análise de regressão relacionando a porcentagem de germinação de sementes e o comprimento de plântulas em diferentes níveis de potencial osmótico nos solutos manitol e $\mathrm{NaCl}$ pelo teste de incubação em papel de filtro.
FIG. 3 - Análise de regressão relacionando o índice de velocidade de crescimento micelial (IVCM) de Botryodiplodia theobromae (A) e Colletotrichum gossypii var. cephalosporioides (B) em diferentes níveis de potencial osmótico nos solutos manitol e $\mathrm{NaCl}$. o crescimento micelial desse fungo foi maior em meio de cultura modificado, osmoticamente, com sacarose do que em meios de cultura modificados com $\mathrm{NaCl}, \mathrm{KCl}$ e a mistura dos sais $\mathrm{NaCl}, \mathrm{KCl}$ e $\mathrm{NaSO}_{4}(5: 3: 2)$, em potenciais osmóticos semelhantes. Os autores atribuíram a utilização da sacarose como fonte adicional de energia pelo fungo e os efeitos tóxicos dos sais utilizados, principalmente em potenciais osmóticos mais negativos, como sendo os fatores responsáveis pelo crescimento diferenciado do fungo.

Os resultados deste trabalho apresentam tendências observadas em estudos realizados por Coutinho (2000), envolvendo Colletotrichum lindemuthianum (Sacc. \& 

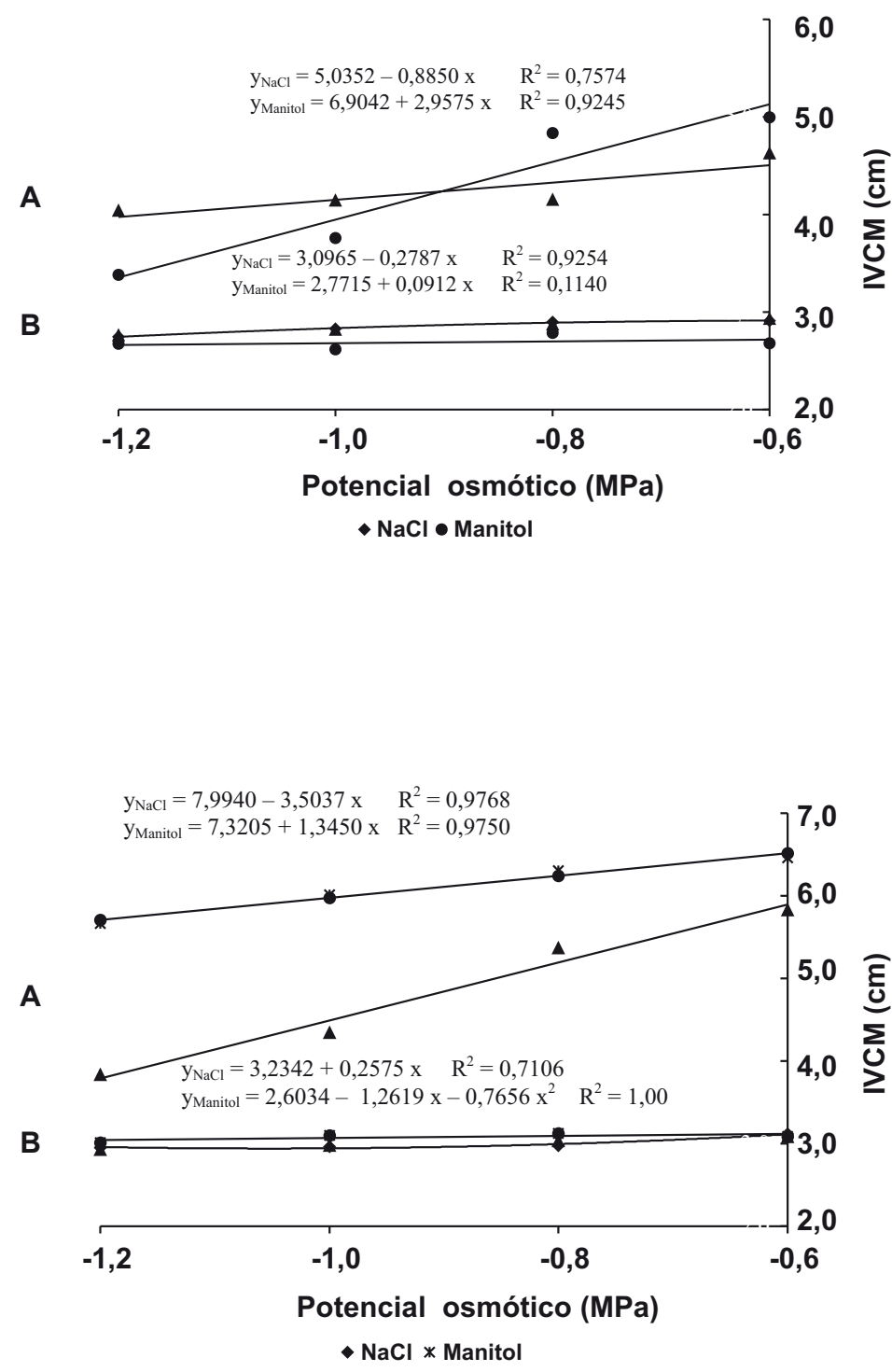

FIG. 4 - Análise de regressão relacionando o índice de velocidade de crescimento micelial (IVCM) de Rhizoctonia solani (A) e Colletotrichum gossypii (B) em diferentes níveis de potencial osmótico nos solutos manitol e $\mathrm{NaCl}$.
FIG. 5 - Análise de regressão relacionando o índice de velocidade de crescimento micelial (IVCM) de Fusarium oxysporum f.sp. vasinfectum (A) e Sclerotinia sclerotiorum (B) em diferentes níveis de potencial osmótico nos solutos manitol e $\mathrm{NaCl}$.
Magnus) Briosi \& Cavara, Fusarium oxysporum f.sp. phaseoli J.B. Kendr. \& W.C. Snyder e R. solani em meio BDA com restrição hídrica induzida pelos solutos manitol, $\mathrm{NaCl}$ e $\mathrm{KCl}$ nos potenciais osmóticos $-0,4 \mathrm{MPa},-0,5 \mathrm{MPa}$, $-0,6 \mathrm{MPa}$ e $-0,7 \mathrm{MPa}$. Nesses estudos, o IVCM dos fungos não foi afetado pela restrição hídrica.

No presente estudo, o crescimento micelial dos fungos B. theobromae, F. oxysporum f.sp. vasinfectum e S. sclerotiorum foi reduzido pela utilização de $\mathrm{NaCl} \mathrm{em}$ comparação ao uso de manitol, indicando um possível efeito tóxico desse sal no crescimento dos fungos. Porém, segundo Coutinho (2000), o maior crescimento micelial dos fungos em meio com manitol pode estar relacionado à utilização desse soluto como fonte adicional de energia ou a um melhor ajuste osmótico da célula fúngica pela absorção dos solutos, permitindo maior turgor para a extensão celular.

Para todos os fungos considerados, não houve efeito de tratamentos sobre a porcentagem de ocorrência desses em sementes de algodoeiro inoculadas, indicando que a restrição hídrica não apresenta efeito negativo no desenvolvimento e deteç̧ão de C. gossypi, C. gossypii var. cephalosporioides, $F$. oxysporum f. sp. vasinfectum e B. theobromae no teste de incubação em substrato de papel (Tabela 2).

Para contornar o rápido desenvolvimento de fungos como B. theobromae nas condições do teste de incubação em substrato de papel, conforme foi observado neste trabalho, é recomendável o emprego de fungicida(s). A adição, por exemplo, de doses baixas de iprodione e de dicloram ao papel de filtro para redução do crescimento de $B$. theobromae e Rhizopus stolonifer (Ehrenb.) Vuill. foi uma alternativa bem sucedida utilizada por Machado \& Langerak (1993). Embora a adição de baixas doses dos fungicidas, nestes casos, não deva afetar os potenciais hídricos do substrato, estudos adicionais nesta direção devam ser realizados. 
TABELA 2 - Porcentagem média de ocorrência de fungos em sementes de algodoeiro inoculadas e submetidas ao teste de sanidade pelo método de incubação em papel de filtro padrão (2,4-D) e papel de filtro com restrição hídrica (manitol e $\mathrm{NaCl},-1,0 \mathrm{MPa}$ )

\begin{tabular}{lcccc}
\hline \hline \multirow{2}{*}{ Tratamentos } & \multicolumn{5}{c}{ Ocorrência (\%) } \\
\cline { 2 - 5 } & $\begin{array}{c}\text { Fusarium oxysporum } \\
\text { f.sp. vasinfectum }\end{array}$ & $\begin{array}{c}\text { Botryodiplodia } \\
\text { theobromae }\end{array}$ & $\begin{array}{c}\text { Colletotrichum } \\
\text { gossypii }\end{array}$ & $\begin{array}{c}\text { C. gossypii var. } \\
\text { cephalosporioides }\end{array}$ \\
\hline $2,4-\mathrm{D}$ & $6,25 \mathrm{a}$ & $24,48 \mathrm{a}$ & $24,45 \mathrm{a}$ & $24,44 \mathrm{a}$ \\
manitol & $15,64 \mathrm{a}$ & $25,00 \mathrm{a}$ & $24,48 \mathrm{a}$ & $24,44 \mathrm{a}$ \\
$\mathrm{NaCl}$ & $13,02 \mathrm{a}$ & $24,48 \mathrm{a}$ & $24,96 \mathrm{a}$ & $23,96 \mathrm{a}$ \\
\hline $\mathrm{CV}(\%)$ & 60,76 & 3,43 & 22,05 & 29,19 \\
\hline
\end{tabular}

Médias seguidas de mesma letra na coluna não diferem entre si pelo teste de Tukey a $5 \%$ de probabilidade.

O emprego da restrição hídrica induzida por manitol e $\mathrm{NaCl}$, nos potenciais osmóticos -0,6 $\mathrm{MPa}$, -0,8 $\mathrm{MPa}$, 1,0 $\mathrm{MPa}$ e -1,2 $\mathrm{MPa}$, reduziu o processo de germinação de sementes e o comprimento de plântulas de algodoeiro nos testes de incubação de forma similar ao 2,4-D (sal de diclorofenoxiacetato de sódio). Em meio ágar (BDA), o procedimento reduziu o crescimento micelial dos fungos estudados em potenciais osmóticos mais negativos que $-0,6 \mathrm{MPa}$. A restrição hídrica produzida por manitol e $\mathrm{NaCl}$ no potencial osmótico $-1,0 \mathrm{MPa}$ não interferiu no desenvolvimento e detecção dos fungos $F$. oxysporum f.sp. vasinfectum, B. theobromae, C. gossypii e C. gossypii var cephalosporioides em sementes infectadas de algodoeiro submetidas ao teste de sanidade pelo método de incubação em substrato de papel.

\section{AGRADECIMENTOS}

Os autores agradecem à Fundação de Apoio à Pesquisa de Minas Gerais - FAPEMIG, ao Conselho Nacional de Desenvolvimento Científico e Tecnológico - CNPq pelo apoio financeiro.

\section{REFERÊNCIAS BIBLIOGRÁFICAS}

ALAM, S., JOYCE, D. \& WEARING, A. Effects of equilibrium relative humidity on in vitro growth of Botrytis cinerea and Alternaria alternata. Australian Journal of Experimental Agriculture 36:383-388. 1996.

AMARAL, E.A.S., VIEIRA, M.G.G.C.\& SANTOS, C.D.Avaliação da influência de diferentes potenciais osmóticos no índice de crescimento de Fusarium moniliforme Sheldon, Aspergillus sp. e Penicillium sp. em meio de cultura. Anais, 15. Seminário Panamericano de Semillas, Gramado, RS e 3. Workshop sobre Marketing em Sementes e Mudas. Gramado RS. 1996. p. 93.

CARVALHO, J.C.B. Uso da restrição hídrica na inoculação de Colletotrichum lindemunthianum em sementes de feijoeiro (Phaseolus vulgaris L.). Dissertação de Mestrado. Lavras MG. Universidade Federal de Lavras. 1999.

CASSETARINETO, D. \& MACHADO, A.Q. Doenças do algodoeiro: Diagnose e Controle. $1^{\text {a }}$ ed. Cuiabá. 2000.

CIA, E. \& SALGADO, C.L. Doenças do algodoeiro (Gossypium spp.). In: Kimati, H., Amorim, L., Bergamin Filho, A., Camargo, L.E.A. \& Rezende, J.A.M. (Eds.) Manual de Fitopatologia: Doenças das plantas cultivadas. 3.ed. São Paulo SP. Agronômica Ceres, v.2, 1997. pp. 33-48.

COSTA, M.L.N. Inoculação de Fusarium oxysporum f.sp. phaseoli em sementes de feijoeiro por meio da restrição hídrica. Dissertação de Mestrado. Lavras MG. Universidade Federal de Lavras. 2000.

COUTINHO, W.M. Uso da restrição hídrica no controle da germinação de sementes de arroz (Oryza sativa L.) e feijoeiro (Phaseolus vulgaris L.) em testes de sanidade. Dissertação de Mestrado. Lavras MG. Universidade Federal de Lavras. 2000.

GAO, S. \& SHAIN, L. Effect of osmotic potential on virulent and hypovirulent strains of the chestnut blight fungus. Canadian Journal of Forest Research 25:1024-1029. 1995.

HEYDECKER, W., HIGGINS, J. \& TURNER, Y.J. Invigoration of seeds? Seed Science and Technology (Zürich) 3:881-888. 1975.

JULIATTI, F.C. \& RUANO, O. Algodão (Gossypium hirsutum L.): Controle de doenças. In: Vale, F.X.R. \& Zambolim, L. (Eds.) Controle de Doenças de Plantas. v. 2, Viçosa MG. UFV. 1997. pp. 555-558.

LIMONARD, T. Ecological aspects of seed health testing. Proceedings of International Seed Testing Association(Wageningen) 33:343-513. 1968.

MACHADO. J.C. Studies on some seed-borne diseases of zinnia, african marigolds and soybean. PhD Thesis. Manchester UK. University of Manchester. 1980.

MACHADO, J.C. \& LANGERAK, C.J. Improvement of a blotter method to detect economically important fungi associated with seeds of cotton. In: Ista Plant Disease Committee Symposium On Seed Health Testing. Ottawa. 1993. pp. 48-58.

MACHADO, J.C., OLIVEIRA, J.A., VIEIRA, M.G.G.C. \& ALVES, M.C. Uso da restrição hídrica na inoculação de fungos em sementes de algodoeiro (Gossypium hirsutum L.) Revista Brasileira de Sementes 26:62-67. 2004a.

MACHADO, J.C., GUIMARAES R.M., VIEIRA, M.G.G.C, SOUZA, R.M. \& POZZA, E.A. Use of water restriction technique in seed pathology. ISTA News Bulletin 128:14-18. 2004b. 
OLIVEIRA, J.A. Efeito do tratamento fungicida em sementes e no controle de tombamento de plântulas de pepino (Cucumis sativus L.) e pimentão (Capsicum annum L.) Dissertação de Mestrado. Lavras MG. Universidade Federal de Lavras. 1991.
POZZA, E.A. \& JULIATTI, F.C. Tratamento de sementes com fungicidas no controle de doenças iniciais do algodoeiro. Fitopatologia Brasileira 19:384-389. 1994.

Recebido 4 Fevereiro 2003 - Aceito 1 Outubro 2007 - FB 3012 